\title{
COMPARISON OF ROUTING STRATEGIES FOR AGV SYSTEMS USING SIMULATION
}

\author{
Mark B. Duinkerken \\ Jaap A. Ottjes \\ Gabriel Lodewijks \\ Faculty of Mechanical, Maritime and Materials Engineering \\ Delft University of Technology \\ Mekelweg 2 \\ 2628 CD Delft, THE NETHERLANDS
}

\begin{abstract}
In automated transport systems, the origin-destination combinations are normally connected through a fixed layout, not representing the shortest path. The flexibility of these systems is limited and often the infrastructure is not optimally used. With the introduction of more powerful onboard computers and advanced sensor technology, the positioning and navigating possibilities of AGVs increased. However the routes, although virtual, are still fixed. A new step ahead would be to determine each path dynamically. This would use the free ranging capacities of AGVs to its full potential. In this paper, the benefits of the dynamic free ranging approach are investigated; a simulation model on the strategic level is presented that compares several common fixed layouts with the shortest connection approach. Naturally, the avoidance of collisions plays a central role. It is concluded that dynamic free ranging has high potential in terms of transport capacity of the resulting system.
\end{abstract}

\section{PROBLEM FIELD OVERVIEW}

\subsection{AGVs for material handling systems}

In material handling systems, the use of Automated Guided Vehicles (AGVs) is increasing, for more than several decades (Hammond 1986). AGV systems have found wide application in many areas: e.g. warehousing, flexible manufacturing systems and transport of containers on a container terminal. In a large number of today's modern manufacturing and transport systems, AGV systems are the material handling system of choice because of the general belief that they increase productivity, product quality and system flexibility and thus lower costs. However, the design of these systems is complex due to the interrelated decisions that must be made and the large number of system design alternatives that are available.
The design of the AGV system can dramatically affect the cost and quality of a material handling system.

When designing an AGV system, both physical elements such as the vehicles and the facility layout as well as the operational control of the equipment have to be developed. This operational procedure design includes the definition of procedures for dispatching, scheduling and routing. Dispatching is the process of assigning a transportation job to an $\mathrm{AGV}$, where scheduling is the process of dispatching a set of AGVs to a batch of transportation jobs. Routing is the process of determining routes for a set of AGVs to fulfil their respective transportation jobs. In current systems, AGVs use a map of predefined, fixed paths that are combined to obtain routes along which they move from origin to destination point.

Configuration of the guide path for an AGV system involves issues as the location of pickup and delivery points, the path layout and the path types. The distribution of the pickup and delivery points over the infrastructure is very important as they significantly influence the traffic intensity on that infrastructure and the number and length of the possible routes. In most cases the pickup and delivery points are fixed at the location of transfer equipment or workstations. In some situations, the location of pickup and delivery points may be dynamic during operation, e.g. quay cranes on a container terminal. The design of guide path layout is based on the objective functions to be fulfilled. Different objectives can be found in the literature such as minimizing total transport cost of the path, minimizing travel distance or minimizing travel time (Seifert 1995).

\subsection{Fixed paths for AGVs}

The set of fixed routes of an AGV system can be modelled as a graph consisting of nodes connected by a set of arcs. The nodes represent key locations in the AGV system such as pickup and delivery points. The arcs connect- 
ing these nodes represent segments of physical or virtual guide paths to be followed by the AGV. This graph is the primary input to the routing function of the AGV control system. Given the location of an AGV and its prescribed destination, the vehicle router can find the sequence of nodes that specify the path of the vehicle. The objective of the guide path design is to minimize the time or distance the AGV has to travel to complete assignments. The guide path design affects also vehicle requirements and space utilization.

Traditionally, the guide path of an AGV system itself was defined by the (magnetic or optical) guidance system. The facility layout directly affects the guide path design as well as the location of the pick-up and delivery points. The guide path design, in combination with the dispatching rules, directly affects the operational performance: a poor design may cause vehicle congestion or guide path blocking.

\subsection{Container terminal layout}

In Rotterdam, on the container terminal of Europe Container Terminals (ECT), an AGV system is used for the transport of containers between quay and stack area. Figure 1 shows a typical layout for a terminal that can handle two large container vessels at a time (Duinkerken and Ottjes 2000). The two vessels are shown at the bottom of the figure; each vessel is being served by four quay cranes. Containers in the stack are oriented perpendicular to the quay. Containers on the vessel are oriented parallel to the quay. The areas shaded in grey are the operational areas of the quay cranes. The hold covers of the vessels occupy some space in these areas, but mostly the area is kept free to allow the quay cranes to move freely along the quay. Pick up and delivery points in this system are located at the quay cranes and at the long side of the stack.

For AGV routing, it means that all routes to and from the quay cranes lead along the entire length of an operational area. The general setup of the AGV routes will look like the arrows indicated in Figure 1. In reality, the directions consist of several parallel tracks that can be followed by the AGVs. The downside of these parallel tracks is the fact that it is unavoidable that the AGVs will have to cross each others paths. When an AGV makes a ninety degree turn, parallel lanes at the inside and outside will be temporarily blocked. Since the paths of the AGVs are determined in advance and AGVs cannot deviate from their track, nearby AGVs on parallel lanes will have to come to a stop and wait for the AGV to complete its turn. If, for some reason, a deadlock occurs in the AGV-system, the AGVs are unable to solve this deadlock themselves, because they will keep trying to follow the path they were instructed to follow.

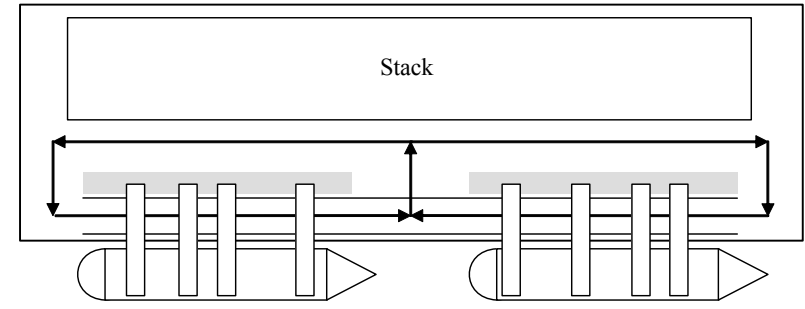

Figure 1: Typical Container Terminal Layout

Although AGV navigation systems enable AGV paths to be quite complex, the routing is mostly still done by the central traffic computer, which determines in advance which route is going to be driven. Every few seconds, this computer will give the AGV a signal that it is allowed to drive the next few meters. This is a very safe system, since a failure in the radio communication system will cause the AGVs to stop within a few meters. However, one would say that a more flexible and intelligent system could be a lot more efficient than the current system (van Dam 2004).

\subsection{Model for Dynamic Evasive Free-ranging Trajectories}

Since the opening of the automated container terminal of ECT, Rotterdam in 1993, several projects has been carried out to improve the performance of such a system. In (Möhring 2004) a network layout is studied where the average travel distance is much shorter compared to the original loop layout. In Evers (1998), an improved layout is proposed with even shorter travel distances (see Figure 2). Still, these systems are based on a fixed layout.

Instead of defining a fixed layout, dynamic routing will determine a trajectory for each new transportation job. As a starting point, a simulation model for the behaviour of pedestrians called NOMAD is used (Hoogendoorn and Bovy 2002, 2003). In that approach the behaviour of pedestrians, choosing their path within an infrastructure is described by modelling pedestrians as economic individuals, optimizing their costs. The model was successfully used for the design of public spaces such as airport terminals and train stations.

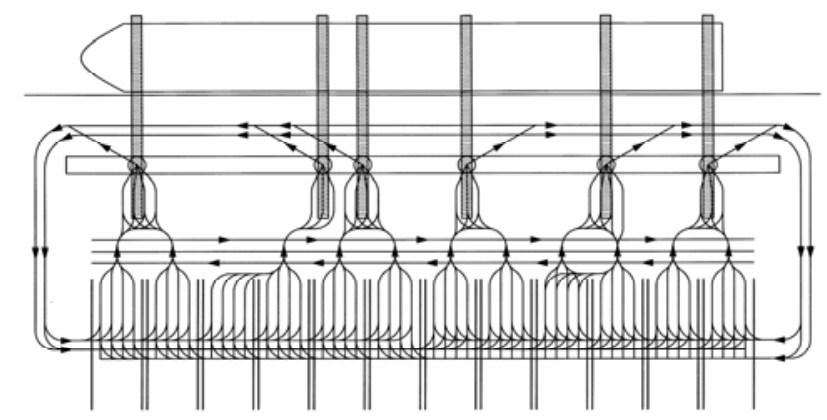

Figure 2: Cross-Over Layout by Evers (1998) 
The new approach for dynamic routing contains three layers, a strategic, a tactical and an operational level. On the tactical level, the model calculates a unique trajectory each time a transportation job arises, thus dynamic. At the operational level, a controller directs an AGV according to this pre-planned trajectory, but avoiding collisions with obstacles, hence evasive. Both levels use the free ranging capacity of the vehicles. The model is based on minimizing costs. Travel time is one of the cost components and therefore the model will tend to minimize the transport distance between origin and destination, resulting in a cross-over behaviour. The new model is called DEFT, which stands for "Dynamic, Evasive, Free-range Trajectories”.

\subsection{Goal of this research}

Papers discussing the operational controller (Duinkerken 2005) and the tactical planning (Duinkerken 2006) of DEFT have been published. However, beside the question whether the DEFT model is feasible, it needs also to be determined whether or not an AGV system with this type of control outperforms the more traditional types of control. Simulating the AGV transport system will increase insight in the use of this approach. This paper presents a simulation model to study the connection between origin and destination points on a terminal at a strategic level. The research goal is to compare the theoretical capacity of terminals using fixed routing with terminals applying a linear cross-over approach.

The remainder of this paper is organized as follows. In the next chapter, a short overview of existing literature is given. Two most used routing strategies are introduced and the cross-over strategy is presented. In chapter 3, more details on the simulation model is given. In chapter 4 experiments and results are discussed and the final chapters contain conclusions and recommendations for further work.

\section{AGV ROUTING AND SCHEDULING}

\subsection{Literature overview}

Research papers on the use of AGVs in automated material handling systems are widely available. Most work study the application of a fixed topology (Ganesharajah 1998). Loop, tandem and network layouts are regarded (Oboth 1999). Research focuses on choice of pickup and delivery points, topology design (Bordelon Hoff and Sarker 1998) and operation research methods for route optimization (Seifert et. al 1995; Möhring 2005).

Besides the industrial application of AGVs, a lot of work is done in the field of robotics, where AGVs are studied as mobile robots (van Turenhout 1994). Time optimal trajectories for car-like vehicles were subject of study for over fifty years (Dubins 1957). With path plan- ning, only the dimensional constraints are taking into account; this problem is also known as the piano movers' problem (Schwartz and Sharir 1983). Trajectory planning takes also time into account. Relevant is whether the nonholonomic constraints are considered as well.

For holonomic planning, exact methods are the roadmap approach using Voronoi diagrams (Takahashi and Schilling 1989), cell decomposition (Latombe 1991) and potential field methods (Khatib 1986). Probabilistic methods use incremental search methods like the randomized potential field approach and the rapidly-exploring random trees method (LaValle 1998).

Trajectory planning with non-holonomic constraints can be done either indirect or direct. Indirect methods try to adapt the results of holonomic methods to the nonholonomic constraints (Laumond 1994; Fraichard and Scheuer 2004). Direct methods integrate the nonholonomic constraints into the trajectory searching method (Barraquand and Latombe 1991).

\subsection{Routing strategies}

For this paper, it is assumed that the location of origin and destination points, the cranes, is fixed. Quaycranes and stacking cranes are on opposite sides of the terminal. Three different trajectory layouts are considered.

The first routing procedure is a procedure where AGVs are routed in a loop. It is illustrated in Figure 3. The average trajectory length will be the sum of the stack length and the terminal width. This topology was originally used at the first automated container terminal in Rotterdam.

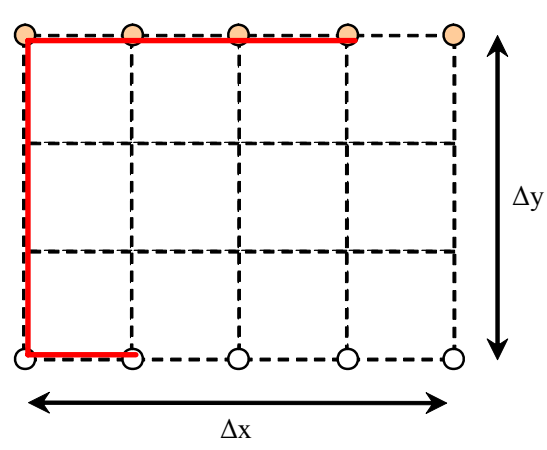

Figure 3: Loop Routing Distance

The second routing procedure is a procedure where AGVs are routed along the shortest route through a mesh. This procedure is illustrated in Figure 4. For an appropriate mesh size, the minimum distance will always be the sum of the absolute difference in $\mathrm{x}$ - and in $\mathrm{y}$-direction $(\Delta \mathrm{x}$ and $\Delta y$ respectively). For comparison purposes, it is assumed that a shortest route can always be driven. This type of topology is used at the automated container terminal in Hamburg (Möhring 2004). 


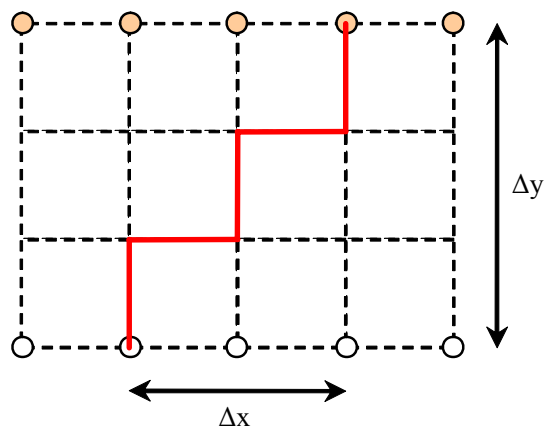

Figure 4: Mesh Routing Distance

The third routing procedure is a procedure where crossover is considered and AGVs are guided along a straight line between origin and destination crane; the shortest possible route (Figure 5). When no obstacles are present and no other $\mathrm{AGV}$ is hindering, there is no need for eva-

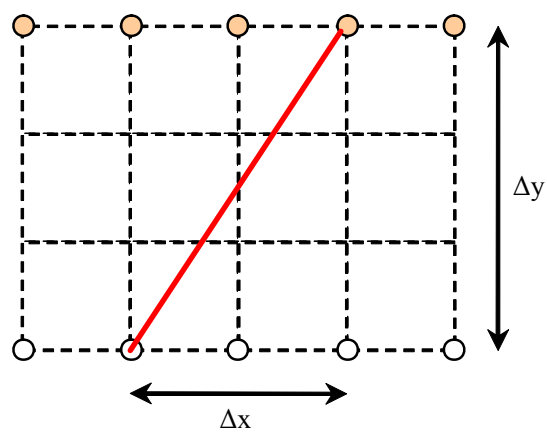

Figure 5: Cross-Over Distance

sive action. This will result in a straight line connection, the shortest possible travel distance. Although it is in fact a fixed path layout, this simplified model is used to study the potential of free-ranging trajectories.

\section{SIMULATION MODEL}

\subsection{Model description}

A discrete event simulation model to compare the performance of different trajectory planning strategies was built, using Delphi in combination with the TOMAS simulation package (Veeke and Ottjes 2002). This model consists of an operational area with quaycranes on one side and stackcranes on the opposite side (see Figure 6).

Transportjobs are from quaycrane to stackcrane and vice versa. For each $\mathrm{AGV}$, a destination is drawn from a uniform distribution. AGVs travel with constant speed. Except for the destination choice, no stochastic behaviour is modelled yet. Begin and end of the trajectories are discarded; no obstacles (like hatch covers) are modelled.

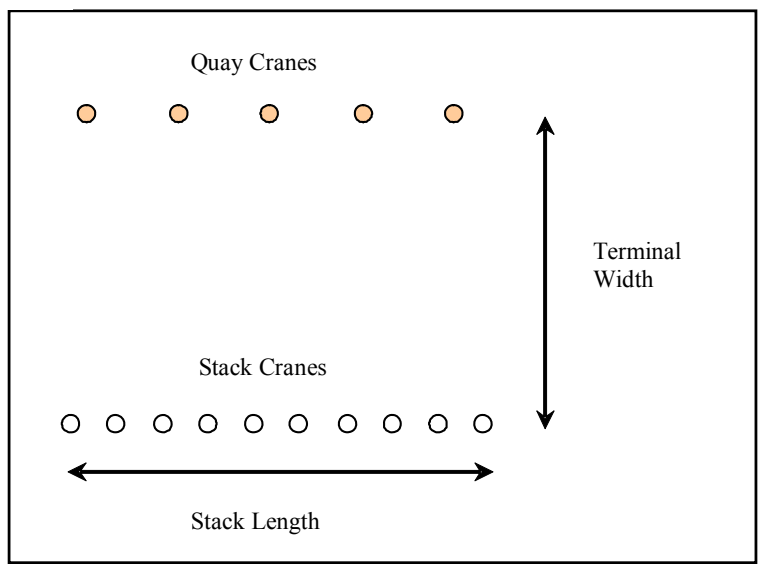

Figure 6: Modelled Layout of the Terminal

\subsection{Trajectory planning}

The distances for the alternative routing algorithms are calculated as favourable as possible. The terminal ends of the loop layout, which in reality are in reality large distances, are neglected. In the loop and mesh cases the influences of curves are neglected and in all cases it is assumed that the shortest route is always available. In the basic scenario, each AGV travels the planned trajectory without considering the other vehicles. The number of collisions can be counted.

A more realistic performance is obtained with a planning that prevents all collisions. Only for the cross-over variant this is modelled. With a heuristic method, for each AGV the earliest starttime that guarantees an unhindered crossing of the terminal is calculated. Both the speed and the trajectory are fixed. A safety time is used for the minimum distance between two crossing trajectories.

The planning uses time windows to calculate the starttime for a safe crossing. Two results are presented in the next chapter: the 'cross close'-variant shows the result with an AGV width of 2.4 meter and a safety time between vehicles of 0.1 second. In a real system, this will probably too close to be safe. The 'cross safe'-variant uses an AGV width of 3.4 meter and a safety time of 5 seconds. Although the second variant offers more safety margin, in both variants the safety is not guaranteed in case of disturbances. Small disturbances will occur frequently f.i. if the motor speed does not exactly match the planned speed or minor differences in steering angles divert the vehicle from its planned trajectory. The proposed DEFT model provides in an operational controller which can handle situations where deviation from the planned trajectory occurs.

\subsection{Collision detection}

A 2D collision detection algorithm, based on TRAVIS (Duinkerken and Terstegge 2001), is implemented. The 
algorithm uses AGV shapes described as polygons, as shown in Figure 7. A collision is detected if the two polygons overlap. This is the case if any of the segments of polygon 1 intersects with any of segments of polygon 2 . The algorithm, working with a 0.05 second time interval, checks for each combination of AGVs whether they collide.

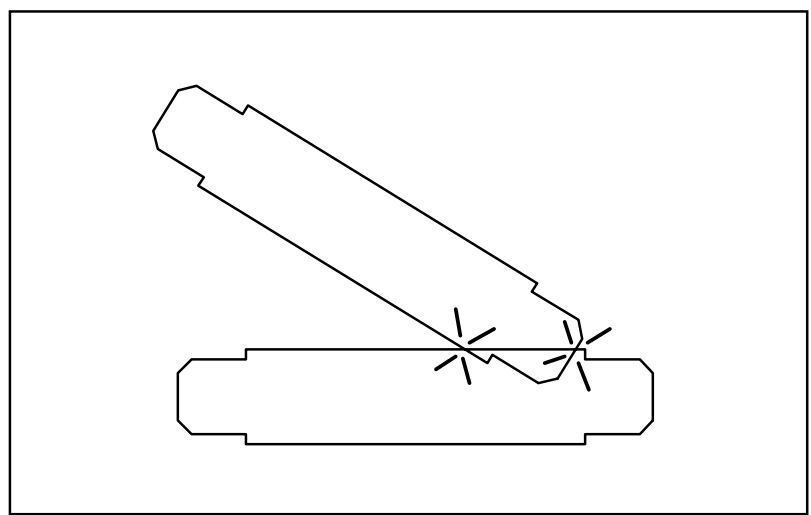

Figure 7: TRAVIS Collision Detection

Distance monitoring is achieved in a similar manner. Instead of checking the intersection of two segments, the distance between two segments is calculated. The distance between two AGVs is the minimum of all the distances between their segments. A screen animation (see Figure 8 ) shows the positions of the AGVs and some basic performance indicators.

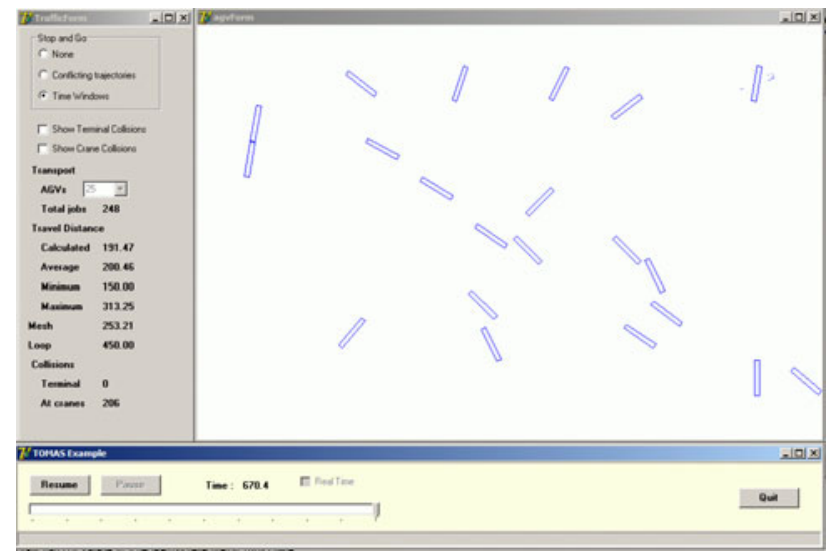

Figure 8: Animation of the Strategic Model

\subsection{Input parameters}

The following input parameters are available in the setup of the simulation model:

- Number of quaycranes and stackcranes

- Quay crane and stack crane handling time distribution (s)
- Quay length, stack length (m) and terminal width (m)

- AGV length, AGV width (m) and AGV speed $(\mathrm{m} / \mathrm{s})$

\section{EXPERIMENTS AND RESULTS}

\subsection{Experimental setup}

All crane handling times in the simulation are set to zero. Thus, only driving AGVs are considered. If handling times are modelled, more AGVs will be needed, although the traffic in the terminal area will be the same. For the mesh and loop strategy, only the average driven distance is used to calculate a possible job performance without taking into account the effects of congestion. Hence, a linear relation between number of $\mathrm{AGVs}$ and transport capacity is assumed. For the cross-strategies, the planning heuristic is used to reduce the number of collisions to zero. Thus, congestion results in non-linear behaviour when large number of AGVs is employed.

\subsection{Results}

In Table 1, the results of the 'cross close' strategy, are presented. The number of AGVs is varied between 1 and 50 . The resulting number of executed transport jobs is almost linear with the number of AGVs below 25. In case more then $25 \mathrm{AGVs}$ are employed the number of executed jobs drops due to congestion effects. In Table 2, the results of the 'cross safe' strategy are shown. It is obvious that the large safety margins cause a faster and larger drop in transport capacity.

In both tables, the number of collisions is given; it is shown that both cross-over variants are safe, as expected. The so-called 'park-conflicts' are the number of occasions that more than one AGV is parked at the same crane. Whether this is a problem or not depends of course on the layout of the terminal.

Table 1 Simulation Results of the 'Cross Close' Variant

\begin{tabular}{|l|rrrrrr|}
\hline AGVs & $\mathbf{1}$ & $\mathbf{5}$ & $\mathbf{1 0}$ & $\mathbf{1 5}$ & $\mathbf{2 0}$ & $\mathbf{2 5}$ \\
\hline jobs / hr & 67 & 361 & 668 & 911 & 1173 & 1330 \\
collisions & 0 & 0 & 0 & 0 & 0 & 0 \\
park-conflicts & 0 & 16 & 151 & 346 & 580 & 966 \\
\hline AGVs & $\mathbf{3 0}$ & $\mathbf{3 5}$ & $\mathbf{4 0}$ & $\mathbf{4 5}$ & $\mathbf{5 0}$ & \\
\hline jobs / hr & 1477 & 1615 & 1766 & 1820 & 1933 & \\
collisions & 0 & 0 & 0 & 0 & 0 & \\
park-conflicts & 1391 & 1833 & 2318 & 2825 & 3469 & \\
\hline
\end{tabular}


Table 2 Simulation Results of the 'Cross Safe' Variant

\begin{tabular}{|l|rrrrrr|}
\hline AGVs & $\mathbf{1}$ & $\mathbf{5}$ & $\mathbf{1 0}$ & $\mathbf{1 5}$ & $\mathbf{2 0}$ & $\mathbf{2 5}$ \\
\hline jobs / hr & 67 & 325 & 533 & 681 & 783 & 820 \\
collisions & 0 & 0 & 0 & 0 & 0 & 0 \\
park-conflicts & 0 & 31 & 169 & 317 & 526 & 785 \\
\hline AGVs & $\mathbf{3 0}$ & $\mathbf{3 5}$ & $\mathbf{4 0}$ & $\mathbf{4 5}$ & $\mathbf{5 0}$ & \\
\hline jobs / hr & 872 & 921 & 961 & 986 & 1029 & \\
collisions & 0 & 0 & 0 & 0 & 0 & \\
park-conflicts & 1169 & 1336 & 1658 & 2038 & 2425 & \\
\hline
\end{tabular}

In Table 3, the average transport distance for the 3 variants is shown. It is clear that the average distance for a transportjob is much shorter for the cross-over variants. Hence, less AGVs are needed or, with equal amount of AGVs, the capacity of cross-over variants is much higher.

Table 3 Average Transport Distances (m)

\begin{tabular}{|l|c|}
\hline layout variant & $\begin{array}{c}\text { transport } \\
\text { distance } \\
(\mathbf{m})\end{array}$ \\
\hline mesh & 253.21 \\
loop & 450.00 \\
cross-over & 191.47 \\
\hline
\end{tabular}

In Figure 9, the relation between the number of AGVs and the performance of the terminal is given. This performance is measured in the number of transportjobs completed. Because in the loop and mesh variants the collision avoidance is not considered, the capacity is linear with the number of AGVs. In the cross-over variants the capacity will drop below the other variants when the number of AGVs is too high; the advantage of shorter distances is annihilated because of the density of AGVs on the terminal. However, when collision avoidance is implemented for the mesh and loop variants, this effect will also occur.

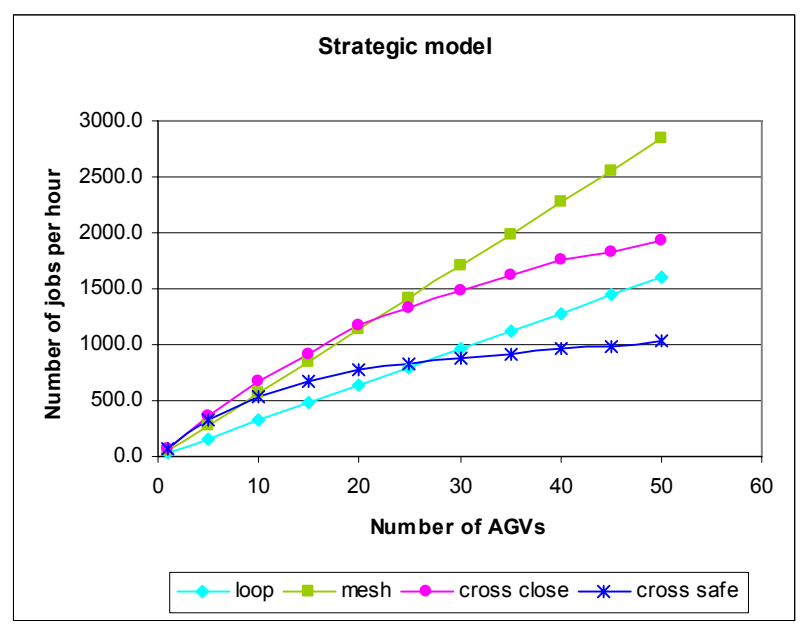

Figure 9 Performance of the Different Routing Strategies

\section{CONCLUSIONS}

The strategic model for evaluating layout variants was implemented successfully. A useful comparison between the methods was made. A realistic scenario of cross-over, including collision avoidance, is compared with the bestcase variants of traditional layouts (ignoring congestion effects). In the basic setup, where terminal dimensions resemble existing terminals, the cross-variants outperform the loop variant when less then $25 \mathrm{AGVs}$ are used. With less then $20 \mathrm{AGVs}$, the cross variant is better than the mesh-variant.

For a high performance container terminal, 60 moves per hour per quaycrane are needed. The terminal in this model uses 6 quaycranes, so 360 moves per hour means 720 transportjobs. From the results it can be concluded that about 10 to 15 active AGVs in the terminal area are sufficient. Of course, this number represents only the AGVs in the transport area of the terminal because beginand end-trajectory, as well as handling times are neglected.

Furthermore, the influence of the safety margins in the cross-over strategy was shown. With more experiments the influence of the length and width of the terminal is studied, as well as the effects of other important parameters as the crane handling times and the size and shape of the vehicles.

\section{FURTHER DEVELOPMENTS}

Collision avoidance for the mesh and loop variants is not considered in this paper. From previous work (Duinkerken 2000) it is known that congestion is a major bottleneck for terminals with either loop or mesh-layout. With implementation of safety procedures for these variants a more realistic performance can be calculated. Because safety will reduce the capacity of loop and mesh variants, the comparison will be in the advantage of crossover variants.

Whether cross-over is a feasible solution for terminal transport must be studied further. More work on the tactical and operational controllers of DEFT is pending. Implementation of the tactical layer of DEFT in the simulation model presented in this paper will result in more accurate estimation of the terminal transport capacity. Study of the operational layer will determine the optimal size for the safety margins.

No stochastic behaviour is modelled in the model, except for the destination choice. However, discrete event simulation is an excellent tool to model and study the influence of stochastic processing times for crane handling, navigation systems, communication delays, etcetera on the performance of the overall terminal system.

The goal of the research project is improving the capacity and accuracy of an automated transport system. For 
this, the DEFT model consists of a strategic, tactical and operational layer. The combination of tactical controller with operational controller has to be tested. After that, the controllers can be integrated with a strategic controller. A laboratory using the complete DEFT model for the study of a scale version of an automated container terminal is under development (see Figure 10).

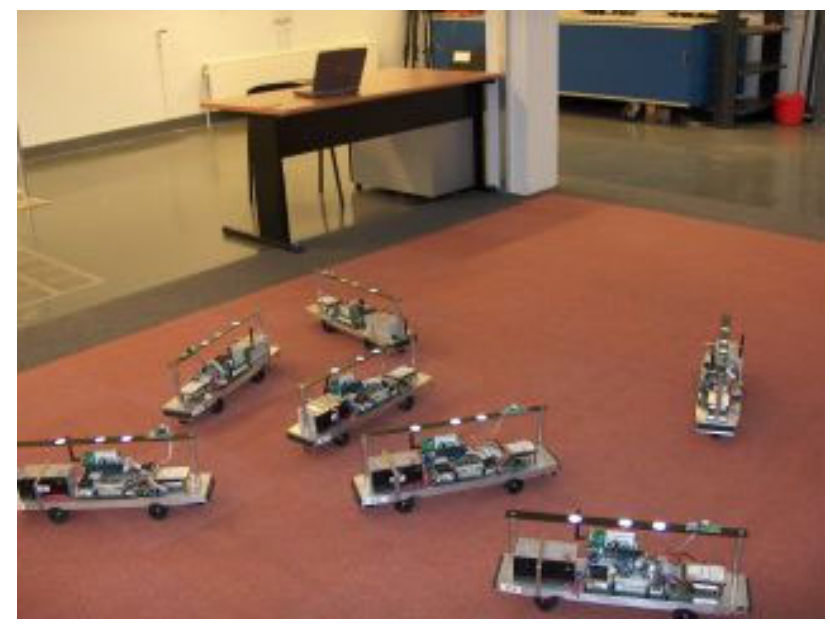

Figure 10 AGV Laboratory for the Study of Automated Transport Systems

\section{ACKNOWLEDGMENTS}

This work was supported in part by the Next Generation Infrastructures Foundation.

\section{REFERENCES}

Barraquand, J. and J.C. Latombe. 1991. Nonholonomic multibody mobile robots: Controllability and motion planning in the presence of obstacles. In IEEE International Conference on Robotics \& Automation, 2328-2335.

Bordelon Hoff, E. and B.R. Sarker. 1998. An overview of path design and dispatching methods for automated guided vehicles. In Integrated Manufacturing Systems, Vol. 9 No. 5, 296-307.

Dam, K.H. van, J.A. Ottjes, G. Lodewijks, Z. VerwaterLukszo and R.W. Wagenaar. 2004. Intelligent Infrastructures: Distributed Intelligence in Transport System Control - an Illustrative Example. In: Proceedings of the International Conference on Systems, Man and Cybernetics. IEEE, The Hague, 4650 - 4654.

Dubins, L.E. 1957. On Curves of Minimal Length with a Constraint on Average Curvature, and with Prescribed Initial and Terminal Positions and Tangents. In: American Journal of Mathematics, Vol. 79, No. 3, 497-516.

Duinkerken, M.B. and J.A. Ottjes. 2000. A simulation model for automated container terminals. In : Pro- ceedings of the Business and Industry Simulation Symposium. Washington D.C. [SCS], 134-139.

Duinkerken, M.B. and M.J. Terstegge. 2001. TRAVIS : an engineering tool to animate and validate AGVsystems. In: Proceedings of the International Conference on Simulation and Multimedia in Engineering Education. Phoenix [SCS].

Duinkerken, M.B., A.J.G. Nuttall, J.A. Ottjes and G. Lodewijks. 2005. Developing an AGV motion controller using simulation, emulation and prototyping. In: IEEE Intelligent Vehicles Symposium Proceedings. Las Vegas NV, USA.

Duinkerken, M.B., M. van der Zee and G. Lodewijks. 2006. Dynamic free range routing for Automated Guided Vehicles. In: IEEE International Conference on Networking, Sensing and Control. Ft. Lauderdale.

Evers, J.J.M., G.J.M. van der Wielen, J.A. Ottjes and M.B. Duinkerken. 1998. The jumbo container terminal: quay transport during ship loading. TRAIL Studies, Delft University of Technology.

Ganesharajah, T., N.G. Hall and C. Sriskandarajah. 1998. Design and operational issues in AGV-served manufacturing systems. In: Annals of Operations Research, Vol. 76, Issue. 0, 109 - 154.

Hammond, G. 1986. AGVS at work, Automated Guided Vehicle Systems. Luton: Bartham Press Ltd.

Hoogendoorn, S.P. and P.H.L. Bovy. 2002. Pedestrian route-choice and activity scheduling theory and methods. In: Transportation Research B. Vol. 38, No. 2, 169-190.

Hoogendoorn, S.P. 2003. Pedestrian Travel Behavior Modeling. In: Proceedings of the 10th international Conference on Travel Behavior Research, Lucerne.

Khatib, O. 1986. Real-Time Obstacle Avoidance for Manipulators and Mobile Robots. In: The International Journal of Robotics Research. 90-98.

Latombe, J.C. 1991. Robot Motion Planning.

Laumond, J.P., P.E. Jacobs, M. Taix and R.M. Murray. 1994. A Motion Planner for Nonholonomic Robots. In: IEEE Transaction on Robotics and Automation; Vol. 10, No.5, 577-593.

LaValle, S.M. 1998. Rapidly exploring random trees: A new tool for path planning. Report number TR.98-11, Iowa State University.

Möhring, R.H., E. Köhler, E. Gawrilow and B. Stenzel. 2004. Conflict-free Real-time AGV routing. In: $O p$ erations Research Proceedings.

Oboth, C., R. Batta and M. Karwan. 1999. Dynamic conflict-free routing of automated guided vehicles. In: International Journal of Production Research, Vol. 37, No. 9, 2003-2030.

Schwartz, J.T. and M. Sharir. 1983. On the piano movers problem: I. the case of a two-dimensional rigid polygonal body moving amidst polygonal barriers. In: 
Communications on pure and applied mathematics. Vol. 36, 345-398.

Seifert, R.W., M.G. Kay and J.R. Wilson. 1995. Routing Strategies using hierarchical simulation. In: Proceedings of the 1995 Winter Simulation Conference.

Takahashi, O. and R.J. Schilling. 1989. Motion planning in a plane using generalized Voronoi diagrams. In:
IEEE Transactions on Robotics and Automation. Vol. 5, No. 2, 143-150.

Turennout, P. van. 1994. Autonomous motion on wheels. Veeke, H.P.M. and J.A. Ottjes. 2002. TOMAS: Tool for Object-Oriented Modelling and Simulation. In: Proceedings of the Business and Industry Simulation Symposium. Washington D.C. 